\title{
La "version ondelettes" du théorème du Jacobien
}

\author{
Sylvia Dobyinsky
}

Résumé. Nous définissons un "produit renormalisé" par ondelettes qui améliore, dans certains cadres fonctionnels, les propriétés du produit usuel de deux fonctions. Grâce à cette technique de renormalisation du produit nous obtenons une démonstration par ondelettes d'une version précisée du théorème du Jacobien. Finalement nous établissons le lien entre ce produit renormalisé par ondelettes et les paraproduits de J.M. Bony.

\section{Introduction.}

Certaines opérations algébriques bilinéaires, telles que le produit de deux distributions, deviennent impossibles dans des cadres fonctionnels inadéquats. Pour contourner cette difficulté, il convient, soit de modifier la définition de l'opération bilinéaire, soit d'ajuster le cadre fonctionnel. Nous définissons donc, à partir de la décomposition dans une base orthonormée d'ondelettes de deux fonctions $f, g \in L^{2}$, des opérateurs bilinéaires qui généralisent et améliorent le produit usuel, que nous appellerons opérateurs de "produit renormalisé". Dans ce texte, nous travaillerons dans des cadres fonctionnels du type $L^{p} \times L^{q}$, et nous ferons en sorte que, par exemple, le "produit renormalisé" de deux fonctions de $L^{2}$ fournisse une fonction de l'espace de Hardy $\mathcal{H}^{1}$. Remarquons cependant que "multiplier" une fonction $a \in$ BMO par une fonction $f \in L^{2}$ nécessite une modification du produit usuel qui n'est pas du tout la même que celle que nous envisageons ici. Il n'y a donc pas de solution 
"universelle" au problème de généraliser la définition du produit.

Soulignons surtout le fait que d'autres techniques bien connues, en particulier la théorie de Littlewood-Paley, et les paraproduits de J. M. Bony permettant de "paramultiplier" deux distributions tempérées arbitraires, fournissent des opérateurs de produit renormalisé équivalents à ceux que nous obtenons à l'aide des ondelettes.

On désigne par $\mathcal{H}^{1}\left(\mathbb{R}^{2}\right)$ l'espace de Hardy dans la version définie par E. Stein et G. Weiss: $f$ appartient à $\mathcal{H}^{1}\left(\mathbb{R}^{2}\right)$ si et seulement si $f$ et les transformées de Riesz $R_{1} f$ et $R_{2} f$ appartiennent toutes trois à $L^{1}\left(\mathbb{R}^{2}\right)$. L'appartenance de $f$ à cet espace $\mathcal{H}^{1}\left(\mathbb{R}^{2}\right)$ peut être caractérisée par une condition portant sur les modules des coefficients d'ondelettes de $f, c f .[13]$.

Le théorème du Jacobien [5] est l'énoncé suivant:

Si $f(x, y)$ et $g(x, y)$ sont deux fonctions appartenant à $L_{\mathrm{loc}}^{1}\left(\mathbb{R}^{2}\right)$ et si les quatre dérivées (prises au sens des distributions) $\partial f / \partial x, \partial f / \partial y$, $\partial g / \partial x, \partial g / \partial y$ appartiennent $\grave{a} L^{2}\left(\mathbb{R}^{2}\right)$, alors le Jacobien

$$
J(f, g)=\frac{\partial f}{\partial x} \frac{\partial g}{\partial y}-\frac{\partial g}{\partial x} \frac{\partial f}{\partial y}
$$

appartient à l'espace de Hardy $\mathcal{H}^{1}\left(\mathbb{R}^{2}\right)$.

On observe bien évidemment que $(\partial f / \partial x)(\partial g / \partial y)$ appartient à $L^{1}\left(\mathbb{R}^{2}\right)$, et qu'il en est de même pour $(\partial g / \partial x)(\partial f / \partial y)$. C'est la différence entre ces deux termes qui introduit précisément la cancellation nécessaire pour passer de $L^{1}\left(\mathbb{R}^{2}\right)$ à $\mathcal{H}^{1}\left(\mathbb{R}^{2}\right)$.

Nous allons donner une nouvelle démonstration en même temps qu'une version précisée de ce résultat. Cette démonstration est basée sur la technique de renormalisation par ondelettes du produit de deux fonctions. Elle s'applique également à un cadre fonctionnel un peu plus général que $L^{2} \times L^{2}$, et à des opérateurs bilinéaires plus généraux que le Jacobien, à savoir

$$
B(f, g)=\sum_{i=1}^{K} A_{i}(f) B_{i}(g),
$$

où $A_{i}$ et $B_{i}$ sont des opérateurs d'intégrales singulières du type étudié par A. Calderón et A. Zygmund, liés par des conditions d'oscillation (les hypothèses seront précisées par la suite). 
En appendice nous établissons l'équivalence, "modulo" un espace strictement contenu dans l'espace de Hardy $\mathcal{H}^{1}$, entre le produit renormalisé par ondelettes et le produit renormalisé par paraproduit.

Soulignons finalement que la méthode de renormalisation du produit à l'aide des paraproduits fournit une démonstration du lemme "Div-Curl" qui est "optimale" du point de vue de sa simplicité (voir [5]), même si toutes les preuves mènent à un résultat équivalent.

\section{La renormalisation par ondelettes du produit usuel.}

\subsection{Les ondelettes utilisées.}

Les ondelettes $\left(\psi_{\lambda}\right)_{\lambda \in \Lambda}$, dont nous rappelons ici la construction et les propriétés essentielles, constituent une base orthonormée remarquable de $L^{2}\left(\mathbb{R}^{n}\right)$.

On désigne par $\Lambda$ l'ensemble des points $\lambda=(k+\varepsilon / 2) 2^{-j}$ où $j \in \mathbb{Z}$, $k \in \mathbb{Z}^{n}$ et $\varepsilon \in E=\{0,1\}^{n} \backslash\{(0,0, \ldots, 0)\}$.

La base orthonormée d'ondelettes $\left(\psi_{\lambda}\right)_{\lambda \in \Lambda}$ est engendrée à partir de $2^{n}-1$ "ondelettes-mère", que nous notons $\left(\psi^{\varepsilon}\right)_{\varepsilon \in E}$ de la façon suivante

$$
\psi_{\lambda}(x)=2^{n j / 2} \psi^{\varepsilon}\left(2^{j} x-k\right) \quad \text { si } \quad \lambda=\left(k+\frac{\varepsilon}{2}\right) 2^{-j} .
$$

On demande à chacune de ces fonctions $\psi^{\varepsilon}$ de satisfaire aux propriétés suivantes:

(1.2) régularité: $\psi^{\varepsilon}$ de classe $C^{s}$ pour un certain $s \geq 1$;

(1.3) localisation:

$$
\left|\partial^{\alpha} \psi^{\varepsilon}(x)\right| \leq C_{m}(1+|x|)^{-m}
$$

pour tout multi-indice $\alpha$ tel que $|\alpha| \leq s$, tout entier $m \geq 1$

et tout $x \in \mathbb{R}^{n}$;

(1.4) $\left(\psi_{\lambda}\right)_{\lambda \in \Lambda}$ est une base orthonormée de $L^{2}\left(\mathbb{R}^{n}\right)$.

Des propriétés précédentes découle alors celle de cancellation

$$
\int_{\mathbb{R}^{n}} x^{\beta} \psi^{\varepsilon}(x) d x=0, \quad \text { si } \quad 0 \leq|\beta| \leq s .
$$


La construction d'une base orthonormée d'ondelettes repose sur le concept d'analyse multirésolution introduit par S. Mallat et Y. Meyer ([11] et [12]). Rappelons tout d'abord la définition d'une analyse multirésolution dans le cas unidimensionnel.

Définition 1.1. Une analyse multirésolution de $L^{2}(\mathbb{R})$ est une suite $\left(V_{j}\right)_{j \in \mathbb{Z}}$ de sous-espaces vectoriels fermés de $L^{2}(\mathbb{R})$, vérifiant les propriétés suivantes:

$$
\begin{gathered}
V_{j} \subset V_{j+1} \\
f(x) \in V_{j} \quad \text { si et seulement si } f(2 x) \in V_{j+1} \\
\bigcap_{j \in \mathbb{Z}} V_{j}=\{0\} \\
\bigcup_{j \in \mathbb{Z}} V_{j} \quad \text { est dense dans } L^{2}(\mathbb{R}) \\
i l \text { existe une fonction } \varphi(x) \in V_{0} \text { telle que } \\
\{\varphi(x-k)\}_{k \in \mathbb{Z}} \text { soit une base hilbertienne de } V_{0} .
\end{gathered}
$$

Considérons maintenant $W_{j}$, le supplémentaire orthogonal de $V_{j}$ dans $V_{j+1}$. On a

$$
\begin{gathered}
L^{2}(\mathbb{R})=\bigoplus_{j \in \mathbb{Z}}^{\perp} W_{j} \\
f(x) \in W_{0} \text { si et seulement si } f\left(2^{j} x\right) \in W_{j} .
\end{gathered}
$$

La construction d'une base orthonormée d'ondelettes de $L^{2}(\mathbb{R})$ revient finalement à celle d'une base de $W_{0}$ de la forme $(\psi(x-k))_{k \in \mathbb{Z}}$. La construction de cette fonction $\psi$ se fait automatiquement à partir de $\varphi($ voir $[13])$.

Revenons maintenant au cas multidimensionel. On pose, pour $\varepsilon=$ $\left(\varepsilon_{1}, \ldots, \varepsilon_{n}\right) \in E$,

$$
\psi^{\varepsilon}(x)=\varphi^{\varepsilon_{1}}\left(x_{1}\right) \cdots \varphi^{\varepsilon_{n}}\left(x_{n}\right)
$$


où

$$
\begin{array}{lll}
\varphi^{\varepsilon_{i}}=\varphi, & \text { si } \quad \varepsilon_{i}=0, \\
\varphi^{\varepsilon_{i}}=\psi, & \text { si } \quad \varepsilon_{i}=1 .
\end{array}
$$

Alors, $\left\{2^{n j / 2} \psi^{\varepsilon}\left(2^{j} x-k\right)\right\}_{j \in \mathbb{Z}, k \in \mathbb{Z}^{n}, \varepsilon \in E}$ est une base orthonormée d'ondelettes de $L^{2}\left(\mathbb{R}^{n}\right)$.

Soulignons finalement que nous disposons de bases orthonormées d'ondelettes à support compact, réelles, de régularité $s(1 \leq s<+\infty)$ arbitraire, construites par I. Daubechies [6].

Note. Dans le but de simplifier les notations, nous supposerons par la suite que toutes les fonctions considérées sont à valeurs réelles.

\subsection{La construction du produit renormalisé par ondelettes.}

Notons $P_{j}$ et $Q_{j}$ les opérateurs de projection orthogonale de $L^{2}\left(\mathbb{R}^{n}\right)$ sur $V_{j}$ et $W_{j}$ respectivement. On a alors

$$
\begin{gathered}
\lim _{j \rightarrow+\infty}\left\|P_{j} f-f\right\|_{2}=0, \quad \text { pour tout } f \in L^{2}\left(\mathbb{R}^{n}\right), \\
\lim _{j \rightarrow-\infty} P_{j} f=0 \quad \text { pour tout } f \in L^{2}\left(\mathbb{R}^{n}\right) \\
P_{j+1}=P_{j}+Q_{j} .
\end{gathered}
$$

Les propriétés (1.13) et (1.14) nous permettent d'écrire le produit ponctuel $f g$ de deux fonctions de $L^{2}\left(\mathbb{R}^{n}\right)$ sous la forme d'une série télescopique, convergente dans $L^{1}$ :

$$
f g=\sum_{j \in \mathbb{Z}}\left[\left(P_{j+1} f\right)\left(P_{j+1} g\right)-\left(P_{j} f\right)\left(P_{j} g\right)\right] .
$$

Grâce à (1.15), on obtient

$$
f g=\sum_{j \in \mathbb{Z}}\left(P_{j} f\right)\left(Q_{j} g\right)+\sum_{j \in \mathbb{Z}}\left(Q_{j} f\right)\left(P_{j} g\right)+\sum_{j \in \mathbb{Z}}\left(Q_{j} f\right)\left(Q_{j} g\right) .
$$


Notons maintenant

$$
\begin{aligned}
\pi_{1}(f, g) & =\sum_{j \in \mathbb{Z}}\left(P_{j} f\right)\left(Q_{j} g\right), \\
\pi_{2}(f, g) & =\sum_{j \in \mathbb{Z}}\left(Q_{j} f\right)\left(P_{j} g\right), \\
S(f, g) & =\sum_{\lambda}\left\langle f, \psi_{\lambda}\right\rangle\left\langle g, \psi_{\lambda}\right\rangle \psi_{\lambda}^{2}, \\
\pi_{3}(f, g) & =\sum_{j \in \mathbb{Z}}\left(Q_{j} f\right)\left(Q_{j} g\right)-S(f, g) .
\end{aligned}
$$

Ainsi, $S(f, g)$ regroupe tous les termes du produit dont l'intégrale n'est pas nulle. Notre but étant de définir un opérateur de produit renormalisé $P^{\sharp}$ qui soit borné de $L^{2} \times L^{2}$ dans $\mathcal{H}^{1}$, nous adopterons la définition qui consiste tout simplement à ôter tous les termes dépourvus d'oscillations.

Définition 1.2. Soient $f$ et $g$ deux fonctions de $L^{2}\left(\mathbb{R}^{n}\right)$. Nous appellerons produit renormalisé de $f$ et $g$, associé à la base orthonormée d'ondelettes $\left(\psi_{\lambda}\right)_{\lambda \in \Lambda}$, et nous noterons $P^{\sharp}(f, g)$, la fonction de $L^{1}\left(\mathbb{R}^{n}\right)$ définie par

$$
P^{\sharp}(f, g)=f g-\sum_{\lambda \in \Lambda}\left\langle f, \psi_{\lambda}\right\rangle\left\langle g, \psi_{\lambda}\right\rangle\left|\psi_{\lambda}\right|^{2} .
$$

Les propriétés particulières de la base orthonormée d'ondelettes entraînent alors la

Proposition 1.1. L'opérateur $P^{\sharp}$ est continu de $L^{2}\left(\mathbb{R}^{n}\right) \times L^{2}\left(\mathbb{R}^{n}\right)$ dans l'espace de Hardy réel $\mathcal{H}^{1}\left(\mathbb{R}^{n}\right)$.

Afin de simplifier les notations, supposons que les fonctions $\varphi$ et $\psi$ sont à support compact, et concentrons nous sur le cas de la dimension 1 (la démonstration que nous proposons se généralise immédiatement au cas multidimensionnel).

Dans ce contexte, les produits $\varphi_{j k} \psi_{j, k+m}$ s'annulent dès que l' entier $m$ vérifie $|m|>C_{0}$, où la constante $C_{0}$ ne dépend que de la taille 
des supports des fonctions $\varphi$ et $\psi$. Notons $w_{j k}^{m}$ le produit $\varphi_{j k} \psi_{j, k+m}$. Pour chaque valeur de $m$ fixée, la famille $\left(w_{j k}^{m}\right)_{j, k \in \mathbb{Z}}$, a essentiellement les mêmes propriétés que la base orthonormée d'ondelettes, la seule propriété manquante étant l'orthogonalité.

L'opérateur $\pi_{1}$ a donc pour expression

$$
\pi_{1}(f, g)=\sum_{j \in \mathbb{Z}} \sum_{k \in \mathbb{Z}} \sum_{|m| \leq C_{0}} 2^{j / 2}\left\langle f, \varphi_{j k}\right\rangle\left\langle g, \psi_{j, k+m}\right\rangle w_{j k}^{m} .
$$

Rappellons maintenant la caractérisation par ondelettes de l'espace de Hardy $\mathcal{H}^{1}\left(\mathbb{R}^{n}\right)$ :

$$
\sum_{\lambda \in \Lambda} \alpha_{\lambda} \psi_{\lambda} \in \mathcal{H}^{1}\left(\mathbb{R}^{n}\right) \Longleftrightarrow\left(\sum_{\lambda \in \Lambda}\left|\alpha_{\lambda}\right|^{2} 2^{n j} \chi_{\lambda}(x)\right)^{1 / 2} \in L^{1}\left(\mathbb{R}^{n}\right),
$$

où $\chi_{\lambda}$ désigne la fonction indicatrice du cube dyadique associé à $\lambda$, qui est défini par la condition $2^{j} x-k \in\left[0,1\left[^{n}\right.\right.$.

Des calculs standard montrent que l'opérateur $U: L^{2} \rightarrow L^{2}$, défini par $U\left(\psi_{j k}\right)=w_{j k}$, est un opérateur de Calderón-Zygmund qui vérifie les conditions $U(1)=U^{*}(1)=0$, et est en conséquence borné dans $\mathcal{H}^{1}$ et dans BMO.

Pour obtenir la continuité $L^{2} \times L^{2} \rightarrow \mathcal{H}^{1}$, il suffit donc de montrer que, pour tout $m \in \mathbb{Z},|m| \leq C_{0}$, la série

$$
\sum_{j \in \mathbb{Z}} \sum_{k \in \mathbb{Z}} 2^{j / 2}\left\langle f, \varphi_{j k}\right\rangle\left\langle g, \psi_{j, k+m}\right\rangle \psi_{j k} \in \mathcal{H}^{1}(\mathbb{R}),
$$

ce qui revient à vérifier que

$$
\left(\sum_{j \in \mathbb{Z}} \sum_{k \in \mathbb{Z}} 2^{2 j}\left|\left\langle f, \varphi_{j k}\right\rangle\left\langle g, \psi_{j, k+m}\right\rangle\right|^{2} \chi_{j k}(x)\right)^{1 / 2} \in L^{1}(\mathbb{R}) .
$$

Il suffit maintenant de montrer que la fonction

$$
\left(\sup _{\substack{j, k \\ x \in Q_{j k}}}\left|\left\langle f, 2^{j / 2} \varphi_{j k}\right\rangle\right|\right)\left(\sum_{j \in \mathbb{Z}} \sum_{k \in \mathbb{Z}} 2^{j}\left|\left\langle g, \psi_{j, k+m}\right\rangle\right|^{2} \chi_{j k}(x)\right)^{1 / 2}
$$

appartient à $L^{1}(\mathbb{R})$. 
Or, si nous désignons par $f^{*}$ la fonction maximale de Hardy et Littlewood, nous avons, pour tout $x \in \mathbb{R}$,

$$
\sup _{\substack{j, k \\ x \in Q_{j k}}}\left|\left\langle f, 2^{j / 2} \varphi_{j k}\right\rangle\right| \leq C f^{*}(x) .
$$

Pour conclure, il suffit alors d'appliquer l'inégalité de Hölder et de remarquer que

$$
\left\|f^{*}\right\|_{2}\left\|\left(\sum_{j \in \mathbb{Z}} \sum_{k \in \mathbb{Z}} 2^{j}\left|\left\langle g, \psi_{j, k+m}\right\rangle\right|^{2} \chi_{j k}(x)\right)^{1 / 2}\right\|_{L^{2}} \leq C\|f\|_{2}\|g\|_{2} .
$$

La continuité de l'opérateur $\pi_{2}$ s'établit de manière identique.

Quant à $\pi_{3}(f, g)$, des calculs immédiats montrent en fait son appartenance à un espace strictement contenu dans $\mathcal{H}^{1}\left(\mathbb{R}^{n}\right)$, l'espace de Besov homogène $\dot{B}_{1}^{0,1}\left(\mathbb{R}^{n}\right)$, dont nous rappellerons la définition par la suite.

Proposition 1.2. Si $f_{q} \rightarrow f$ pour la topologie faible $\sigma\left(L^{2}, L^{2}\right)$, et $g_{q} \rightarrow$ $g$ pour la même topologie, alors la suite $P^{\sharp}\left(f_{q}, g_{q}\right)$ converge faiblement vers $P^{\sharp}(f, g)$ au sens de la topologie faible $\sigma\left(\mathcal{H}^{1}, \mathrm{VMO}\right)$.

Rappelons que VMO est la fermeture, pour la norme de BMO, de l'espace vectoriel des fonctions continues et nulles à l'infini. L'espace $\mathcal{H}^{1}$ est le dual de VMO.

Considérons l'exemple de l'opérateur $\pi_{1}$. Sous nos hypothèses, et grâce à la Proposition 1.1, la suite $\left(\pi_{1}\left(f_{q}, g_{q}\right)\right)$ est bornée dans $\mathcal{H}^{1}$. Comme l'opérateur $U: \psi_{j k} \rightarrow w_{j k}$ est borné dans $\mathcal{H}^{1}$, il suffit maintenant de montrer que, pour chaque $|m| \leq C_{0}$, la suite

$$
\sum_{j \in \mathbb{Z}} \sum_{k \in \mathbb{Z}} 2^{j / 2}\left\langle f_{q}, \varphi_{j k}\right\rangle\left\langle g_{q}, \psi_{j, k+m}\right\rangle \psi_{j k}
$$

converge au sens de la topologie faible $\sigma\left(\mathcal{H}^{1}, \mathrm{VMO}\right)$ vers

$$
\sum_{j \in \mathbb{Z}} \sum_{k \in \mathbb{Z}} 2^{j / 2}\left\langle f, \varphi_{j k}\right\rangle\left\langle g, \psi_{j, k+m}\right\rangle \psi_{j k}
$$

Ceci découle immédiatement du lemme suivant 
Lemme 1.1. Soit $\left(h_{q}\right)_{q \in \mathbb{N}}$ une suite bornée dans $\mathcal{H}^{1}(\mathbb{R})$. Alors, $h_{q}$ converge vers $h$ au sens de la topologie $\sigma\left(\mathcal{H}^{1}, \mathrm{VMO}\right)$ si et seulement si $\left\langle h_{q}, \psi_{j k}\right\rangle \rightarrow\left\langle h, \psi_{j k}\right\rangle$ pour tout $j \in \mathbb{Z}$ et tout $k \in \mathbb{Z}$.

Cette équivalence est conséquence du fait que la base orthonormée d'ondelettes $\left(\psi_{\lambda}\right)_{\lambda \in \Lambda}$ constitue une famille totale dans l'espace de Banach VMO.

\subsection{L'équivalence entre les opérateurs $P^{\sharp}$ et $\tilde{P}^{\sharp}$ associés à deux bases orthonormées d'ondelettes.}

Une fois le produit renormalisé $P^{\sharp}$ construit, la question suivante se pose tout naturellement : cette renormalisation $P^{\sharp}(f, g)$ est-elle, en un certain sens, indépendante de la base d'ondelettes choisie pour la construction de $P^{\sharp}$ ?

Pour répondre à cette question, nous introduisons maintenant l'espace de Besov homogène $\dot{B}_{1}^{0,1}\left(\mathbb{R}^{n}\right)([13],[15])$.

Définition 1.3. L'espace de Besov homogène $\dot{B}_{1}^{0,1}\left(\mathbb{R}^{n}\right)$ est le sousespace de $L^{1}\left(\mathbb{R}^{n}\right)$ composé des fonctions $f \in L^{1}\left(\mathbb{R}^{n}\right)$ dont la série dans une base orthonormée d'ondelettes $f=\sum_{\lambda \in \Lambda} \alpha_{\lambda} \psi_{\lambda}$ vérifie

$$
\sum_{\lambda \in \Lambda}\left|\alpha_{\lambda}\right| 2^{-n j / 2}<+\infty
$$

En d'autres termes, la fonction $f$ appartient à $\dot{B}_{1}^{0,1}\left(\mathbb{R}^{n}\right)$ si et seulement si $\sum_{\lambda \in \Lambda}\left|\alpha_{\lambda}\right|\left\|\psi_{\lambda}\right\|_{1}<+\infty$, c'est à dire si la série d'ondelettes de $f$ converge normalement dans $L^{1}\left(\mathbb{R}^{n}\right)$.

Une autre caractérisation de $\dot{B}_{1}^{0,1}$ sera utile par la suite.

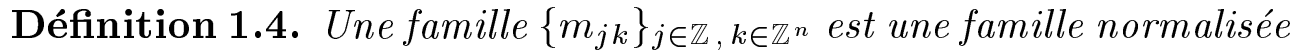
en norme $L^{1}$ de molécules s'il existe deux exposants $\alpha \geq \beta>0$, et une constante $C>0$ tels que

$$
\left|m_{j k}(x)\right| \leq C 2^{n j}\left(1+\left|2^{j} x-k\right|\right)^{-n-\alpha},
$$


pour tout $x \in \mathbb{R}^{n}$,

$$
\left|m_{j k}(x)-m_{j k}(y)\right| \leq C 2^{j(n+\beta)}|x-y|^{\beta}
$$

pour tout $(x, y) \in\left(\mathbb{R}^{n}\right)^{2}$, et

$$
\int_{\mathbb{R}^{n}} m_{j k}(x) d x=0 .
$$

REMARque. Toute base orthonormée d'ondelettes de régularité $s \geq 1$ constitue une famille normalisée en norme $L^{2}$ de molécules.

La deuxième caractérisation de l'espace de Besov homogène $\dot{B}_{1}^{0,1}\left(\mathbb{R}^{n}\right)$ découle du lemme suivant:

Lemme 1.2. Soit $\left(m_{j k}\right)$ une famille normalisée en norme $L^{1}$ de molécules et $\left(\alpha_{j k}\right)$ une suite dans $\ell^{1}\left(\mathbb{Z} \times \mathbb{Z}^{n}\right): \sum_{j} \sum_{k}\left|\alpha_{j k}\right|<+\infty$. Alors $\sum \alpha_{j k} m_{j k}$ appartient $\grave{a} \dot{B}_{1}^{0,1}\left(\mathbb{R}^{n}\right)$.

Nous pouvons maintenant énoncer le

Théorème 1.1. Soient $\left(\psi_{\lambda}\right)_{\lambda \in \Lambda}$ et $\left(\tilde{\psi}_{\lambda}\right)_{\lambda \in \Lambda}$ deux bases orthonormées d'ondelettes de même régularité $s, P^{\sharp}$ et $\tilde{P}^{\sharp}$ les opérateurs de produit renormalisé associés respectivement à chacune de ces bases. Alors l'opérateur $\Delta=P^{\sharp}-\tilde{P}^{\sharp}$ envoie continûment $L^{2}\left(\mathbb{R}^{n}\right) \times L^{2}\left(\mathbb{R}^{n}\right)$ dans $\dot{B}_{1}^{0,1}\left(\mathbb{R}^{n}\right)$.

Cet énoncé signifie que, modulo l'espace $\dot{B}_{1}^{0,1}$, qui est strictement contenu dans $\mathcal{H}^{1}$, toutes les renormalisations du produit par ondelettes d'une certaine régularité sont équivalentes.

Les idées essentielles dans la preuve de ce théorème sont les mêmes que celles qui nous permettront d'établir le théorème du Jacobien. C'est pourquoi nous donnerons ici les étapes principales de cette preuve.

$\mathrm{Si}$

$$
f=\sum_{\lambda} \alpha_{\lambda} \psi_{\lambda}=\sum_{\lambda} \tilde{\alpha}_{\lambda} \tilde{\psi}_{\lambda}
$$

et

$$
g=\sum_{\lambda} \beta_{\lambda} \psi_{\lambda}=\sum_{\lambda} \tilde{\beta}_{\lambda} \tilde{\psi}_{\lambda}
$$


alors la différence entre les deux produits renormalisés $\Delta(f, g)$ s'écrit

$$
\Delta(f, g)=P^{\sharp}(f, g)-\tilde{P}^{\sharp}(f, g)=\Delta_{1}(f, g)+\Delta_{2}(f, g)
$$

où

$$
\Delta_{1}(f, g)=\sum_{\lambda} \tilde{\alpha}_{\lambda} \tilde{\beta}_{\lambda}\left(\psi_{\lambda}^{2}-\tilde{\psi}_{\lambda}^{2}\right)
$$

et

$$
\Delta_{2}(f, g)=\sum_{\lambda}\left(\alpha_{\lambda} \beta_{\lambda}-\tilde{\alpha}_{\lambda} \tilde{\beta}_{\lambda}\right) \psi_{\lambda}^{2} .
$$

Il est facile de vérifier que le terme $\Delta_{1}(f, g)$ appartient à l'espace $\dot{B}_{1}^{0,1}\left(\mathbb{R}^{n}\right)$ car $\left(\psi_{\lambda}^{2}-\tilde{\psi}_{\lambda}^{2}\right)_{\lambda \in \Lambda}$ constitue une famille normalisée en norme $L^{1}$ de molécules, et la suite $\left(\tilde{\alpha}_{\lambda} \tilde{\beta}_{\lambda}\right)_{\lambda \in \Lambda}$ appartient à $\ell^{1}(\Lambda)$. Observons, en effet, que

$$
\int\left(\psi_{\lambda}^{2}-\tilde{\psi}_{\lambda}^{2}\right)=\left\|\psi_{\lambda}\right\|_{2}^{2}-\left\|\tilde{\psi}_{\lambda}\right\|_{2}^{2}=0
$$

La vérification des autres propriétés des molécules est immédiate.

Pour étudier le terme $\Delta_{2}(f, g)$, on remarque que les suites $\left(\alpha_{\lambda}\right)$, $\left(\tilde{\alpha}_{\lambda}\right)$ puis $\left(\beta_{\lambda}\right),\left(\tilde{\beta}_{\lambda}\right)$ sont liées par les équations

$$
\left\{\begin{array}{l}
\tilde{\alpha}_{\lambda}=\sum_{\lambda^{\prime} \in \Lambda} \mu\left(\lambda^{\prime}, \lambda\right) \alpha_{\lambda^{\prime}} \\
\tilde{\beta}_{\lambda}=\sum_{\lambda^{\prime} \in \Lambda} \mu\left(\lambda^{\prime}, \lambda\right) \beta_{\lambda^{\prime}}
\end{array}\right.
$$

où $\mu\left(\lambda^{\prime}, \lambda\right)=\left\langle\tilde{\psi}_{\lambda}, \psi_{\lambda^{\prime}}\right\rangle$.

La matrice de changement de base $M=\left(\mu\left(\lambda^{\prime}, \lambda\right)\right)_{\left(\lambda^{\prime}, \lambda\right) \in \Lambda^{2}}$ est unitaire, et présente une certaine décroissance à partir de la diagonale, que nous détaillons par la suite.

Pour $\gamma>0$, nous définissons les "poids" $p_{\gamma}\left(\lambda, \lambda^{\prime}\right)$ par

$$
p_{\gamma}\left(\lambda^{\prime}, \lambda\right)=\frac{2^{-\left|j^{\prime}-j\right|(\gamma+n / 2)}}{1+\left(j-j^{\prime}\right)^{2}}\left(\frac{2^{-j}+2^{-j^{\prime}}}{2^{-j}+2^{-j^{\prime}}+\left|k 2^{-j}-k^{\prime} 2^{-j^{\prime} \mid}\right|}\right)^{n+\gamma}
$$

Ces $p_{\gamma}\left(\lambda^{\prime}, \lambda\right)$ ont la propriété essentielle suivante (voir [14]) 
Lemme 1.3. Soit $0<\gamma_{3} \leq \gamma_{2} \leq \gamma_{1}$. Il existe une constante $c=$ $c\left(n, \gamma_{1}, \gamma_{2}, \gamma_{3}\right)>0$ telle que

$$
\sum_{\lambda \in \Lambda} p_{\gamma_{2}}\left(\lambda^{\prime}, \lambda\right) p_{\gamma_{1}}\left(\lambda^{\prime \prime}, \lambda\right) \leq c p_{\gamma_{3}}\left(\lambda^{\prime}, \lambda^{\prime \prime}\right)
$$

pour tout $\left(\lambda^{\prime}, \lambda^{\prime \prime}\right) \in \Lambda^{2}$.

Les coefficients de la matrice $M$ sont estimés par

$$
\left|\mu\left(\lambda^{\prime}, \lambda\right)\right| \leq c p_{\gamma_{1}}\left(\lambda^{\prime}, \lambda\right),
$$

pour tout $\left(\lambda^{\prime}, \lambda\right) \in \Lambda^{2}$, où la valeur de $\gamma_{1}$ dépend de la régularité de la base orthonormée d'ondelettes (voir à ce sujet [14]).

Retournons maintenant à $\Delta_{2}(f, g)$ que nous réécrivons

$$
\begin{aligned}
\Delta_{2}(f, g) & =\sum_{\lambda \in \Lambda}\left[\alpha_{\lambda} \beta_{\lambda}-\left(\sum_{\lambda^{\prime}} \alpha_{\lambda^{\prime}} \mu\left(\lambda^{\prime}, \lambda\right)\right)\left(\sum_{\lambda^{\prime \prime}} \beta_{\lambda^{\prime \prime}} \mu\left(\lambda^{\prime \prime}, \lambda\right)\right)\right] \psi_{\lambda}^{2} \\
& =C(f, g)-R(f, g)
\end{aligned}
$$

où

$$
\begin{aligned}
C(f, g) & =\sum_{\lambda \in \Lambda}\left(\alpha_{\lambda} \beta_{\lambda}-\sum_{\lambda^{\prime}} \alpha_{\lambda^{\prime}} \beta_{\lambda^{\prime}} \mu\left(\lambda^{\prime}, \lambda\right)^{2}\right) \psi_{\lambda}^{2} \\
& =\sum_{\lambda \in \Lambda} \alpha_{\lambda} \beta_{\lambda}\left(\psi_{\lambda}^{2}-\sum_{\lambda^{\prime}} \mu\left(\lambda^{\prime}, \lambda\right)^{2} \psi_{\lambda^{\prime}}^{2}\right)
\end{aligned}
$$

et

$$
R(f, g)=\sum_{\lambda} \sum_{\substack{\left.\lambda^{\prime}, \lambda^{\prime \prime}\right) \in \Lambda^{2} \\ \lambda^{\prime} \neq \lambda^{\prime \prime}}} \alpha_{\lambda^{\prime}} \beta_{\lambda^{\prime \prime}} \mu\left(\lambda^{\prime}, \lambda\right) \mu\left(\lambda^{\prime \prime}, \lambda\right) \psi_{\lambda}^{2} .
$$

L'étude de $C(f, g)$ se ramène à celle des fonctions

$$
m_{\lambda}=\psi_{\lambda}^{2}-\sum_{\lambda^{\prime} \in \Lambda} \mu\left(\lambda^{\prime}, \lambda\right)^{2} \psi_{\lambda^{\prime}}^{2}
$$

et découle du

Lemme 1.4. Soit

$$
m_{\lambda}=\psi_{\lambda}^{2}-\sum_{\lambda^{\prime} \in \Lambda} \mu\left(\lambda^{\prime}, \lambda\right)^{2} \psi_{\lambda^{\prime}}^{2}
$$


L'ensemble $\left(m_{\lambda}\right)_{\lambda \in \Lambda}$ constitue alors une famille normalisée en norme $L^{1}$ de molécules.

Dans la preuve du Lemme 1.4, les propriétés de la matrice de passage $M=\left(\mu\left(\lambda^{\prime}, \lambda^{\prime \prime}\right)\right)$ jouent un rôle essentiel.

La série $\sum_{\lambda^{\prime}} \mu\left(\lambda^{\prime}, \lambda\right)^{2}\left\|\psi_{\lambda^{\prime}}^{2}\right\|_{1}$ étant convergente, l'intégration terme à terme donne

$$
\int m_{\lambda}=\int \psi_{\lambda}^{2}-\sum_{\lambda^{\prime}} \mu\left(\lambda^{\prime}, \lambda\right)^{2} \int \psi_{\lambda^{\prime}}^{2}=1-\sum_{\lambda^{\prime}} \mu\left(\lambda^{\prime}, \lambda\right)^{2}=0 .
$$

Les autres propriétés des molécules découlent de la localisation et de la régularité des ondelettes, ainsi que des propriétés particulières des poids $p_{\gamma}\left(\lambda^{\prime}, \lambda\right)$.

Considérons finalement la partie $R(f, g)$, que nous réécrivons

$$
R(f, g)=\sum_{\lambda^{\prime}} \sum_{\lambda^{\prime \prime}} \alpha_{\lambda^{\prime}} \beta_{\lambda^{\prime \prime}} p_{\gamma_{2}}\left(\lambda^{\prime}, \lambda^{\prime \prime}\right) \sum_{\lambda \in \Lambda} \frac{\mu\left(\lambda^{\prime}, \lambda\right) \mu\left(\lambda^{\prime \prime}, \lambda\right)}{p_{\gamma_{2}}\left(\lambda^{\prime}, \lambda^{\prime \prime}\right)} \psi_{\lambda}^{2},
$$

où $\gamma_{2}$ vérifie $0<\gamma_{2}<\gamma_{1}$.

Posons

$$
m_{\lambda^{\prime}, \lambda^{\prime \prime}}=\sum_{\lambda \in \Lambda} \frac{\mu\left(\lambda^{\prime}, \lambda\right) \mu\left(\lambda^{\prime \prime}, \lambda\right)}{p_{\gamma_{2}}\left(\lambda^{\prime}, \lambda^{\prime \prime}\right)} \psi_{\lambda}^{2} .
$$

Alors, si le coefficient $\gamma_{2}$ est choisi convenablement, l'ensemble

$$
\left(m_{\lambda^{\prime}, \lambda^{\prime \prime}}\right)_{\left(\lambda^{\prime}, \lambda^{\prime \prime}\right) \in \Lambda^{2}, \lambda^{\prime} \neq \lambda^{\prime \prime}}
$$

constitue une famille normalisée en norme $L^{1}$ de molécules.

Reste à voir finalement que

$$
\sum_{\lambda^{\prime}} \sum_{\lambda^{\prime \prime}}\left|\alpha_{\lambda^{\prime}}\right|\left|\beta_{\lambda^{\prime \prime}}\right| p_{\gamma_{2}}\left(\lambda^{\prime}, \lambda^{\prime \prime}\right)<+\infty .
$$

L'application du lemme de Schur avec les coefficients $w(\lambda)=2^{-n j / 2}$ à la matrice $\left(p_{\gamma_{2}}\left(\lambda^{\prime}, \lambda^{\prime \prime}\right)\right)_{\left(\lambda^{\prime}, \lambda^{\prime \prime}\right) \in \Lambda^{2}}$ montre que celle-ci définit un opérateur continu dans $\ell^{2}(\Lambda)$ (on pourra consulter [14] à ce sujet).

La série

$$
\sum_{\lambda^{\prime} \in \Lambda} \sum_{\lambda^{\prime \prime} \in \Lambda}\left|\alpha_{\lambda^{\prime}}\right|\left|\beta_{\lambda^{\prime \prime}}\right| p_{\gamma_{2}}\left(\lambda^{\prime \prime}, \lambda^{\prime}\right)
$$

est ainsi majorée par

$$
c\left(\sum_{\lambda^{\prime} \in \Lambda}\left|\alpha_{\lambda^{\prime}}\right|^{2}\right)^{1 / 2}\left(\sum_{\lambda^{\prime \prime} \in \Lambda}\left|\beta_{\lambda^{\prime \prime}}\right|^{2}\right)^{1 / 2}=c\|f\|_{2}\|g\|_{2} .
$$




\section{La "version ondelettes" du théorème du Jacobien.}

Le théorème du Jacobien est l'énoncé suivant [5].

Théorème 2.1. Si $f(x, y)$ et $g(x, y)$ sont deux fonctions appartenant $\grave{a} L_{\mathrm{loc}}^{1}\left(\mathbb{R}^{2}\right)$ et si les quatre dérivées (prises au sens des distributions) $\partial f / \partial x, \partial f / \partial y, \partial g / \partial x, \partial g / \partial y$ appartiennent $\grave{a} L^{2}\left(\mathbb{R}^{2}\right)$, alors le Jacobien

$$
J(f, g)=\frac{\partial f}{\partial x} \frac{\partial g}{\partial y}-\frac{\partial g}{\partial x} \frac{\partial f}{\partial y}
$$

appartient à l'espace de $\operatorname{Hardy} \mathcal{H}^{1}\left(\mathbb{R}^{2}\right)$.

Notre énoncé précisé est le suivant

Théorème 2.2. Soit $\left(\psi_{\lambda}\right)_{\lambda \in \Lambda}$ une base orthonormée d'ondelettes et $P^{\sharp}$ l'opérateur de produit renormalisé associé à cette base. Alors, sous les mêmes hypothèses que le Théorème 2.1 , on a

$$
J(f, g) \in \mathcal{H}^{1}\left(\mathbb{R}^{2}\right)
$$

et

$$
J_{B}(f, g)=J(f, g)-P^{\sharp}\left(\frac{\partial f}{\partial x}, \frac{\partial g}{\partial y}\right)+P^{\sharp}\left(\frac{\partial f}{\partial y}, \frac{\partial g}{\partial x}\right) \in \dot{B}_{1}^{0,1}\left(\mathbb{R}^{2}\right) .
$$

Notre méthode consiste à renormaliser chacun des produits

$$
\frac{\partial f}{\partial x} \frac{\partial g}{\partial y} \quad \text { et } \quad \frac{\partial f}{\partial y} \frac{\partial g}{\partial x}
$$

Les termes $P^{\sharp}(\partial f / \partial x, \partial g / \partial y)$ et $P^{\sharp}(\partial f / \partial y, \partial g / \partial x)$ appartiennent de façon automatique à $\mathcal{H}^{1}\left(\mathbb{R}^{2}\right)$.

Considérons maintenant la somme des "mauvais" termes qui étaient individuellement dépourvus d'oscillations.

$$
J_{B}(f, g)=\sum_{\lambda \in \Lambda}\left(\left\langle\frac{\partial f}{\partial y}, \psi_{\lambda}\right\rangle\left\langle\frac{\partial g}{\partial x}, \psi_{\lambda}\right\rangle-\left\langle\frac{\partial f}{\partial x}, \psi_{\lambda}\right\rangle\left\langle\frac{\partial g}{\partial y}, \psi_{\lambda}\right\rangle\right) \psi_{\lambda}^{2}
$$


$\mathrm{Si}$

$$
f=\sum_{\lambda \in \Lambda} \alpha_{\lambda} \psi_{\lambda}, \quad g=\sum_{\lambda \in \Lambda} \beta_{\lambda} \psi_{\lambda},
$$

l'appartenance à $L^{2}\left(\mathbb{R}^{2}\right)$ des dérivées partielles de $f$ et $g$ entraîne l'appartenance à $\ell^{2}(\Lambda)$ des suites $\left(2^{j} \alpha_{\lambda}\right)_{\lambda \in \Lambda}$ et $\left(2^{j} \beta_{\lambda}\right)_{\lambda \in \Lambda}$.

Posons maintenant

$$
\left\{\begin{array}{l}
\mu_{x}\left(\lambda^{\prime}, \lambda\right)=\left\langle\frac{\partial \psi_{\lambda^{\prime}}}{\partial x}, \psi_{\lambda}\right\rangle \\
\mu_{y}\left(\lambda^{\prime}, \lambda\right)=\left\langle\frac{\partial \psi_{\lambda^{\prime}}}{\partial y}, \psi_{\lambda}\right\rangle
\end{array}\right.
$$

On peut alors écrire

$$
\begin{aligned}
J_{B}(f, g)=\sum_{\lambda \in \Lambda}\left(\sum_{\left(\lambda^{\prime}, \lambda^{\prime \prime}\right) \in \Lambda^{2}} \alpha_{\lambda^{\prime}} \beta_{\lambda^{\prime \prime}}\right. & \left(\mu_{y}\left(\lambda^{\prime}, \lambda\right) \mu_{x}\left(\lambda^{\prime \prime}, \lambda\right)\right. \\
& \left.\left.-\mu_{x}\left(\lambda^{\prime}, \lambda\right) \mu_{y}\left(\lambda^{\prime \prime}, \lambda\right)\right)\right) \psi_{\lambda}^{2} .
\end{aligned}
$$

Remarquons maintenant que l'on a de façon évidente les estimations suivantes

$$
\left|\mu_{x}\left(\lambda^{\prime}, \lambda\right)\right| \leq c 2^{j^{\prime}} p_{\gamma_{1}}\left(\lambda^{\prime}, \lambda\right)
$$

et

$$
\left|\mu_{y}\left(\lambda^{\prime}, \lambda\right)\right| \leq c 2^{j^{\prime}} p_{\gamma_{1}}\left(\lambda^{\prime}, \lambda\right)
$$

où les $p_{\gamma}\left(\lambda^{\prime}, \lambda\right)$ sont définis par l'équation (1.22).

Posons maintenant, pour $0<\gamma_{2}<\gamma_{1}$

$$
m_{\lambda^{\prime} \lambda^{\prime \prime}}=\frac{2^{-j^{\prime}} 2^{-j^{\prime \prime}}}{p_{\gamma_{2}}\left(\lambda^{\prime}, \lambda^{\prime \prime}\right)} \sum_{\lambda \in \Lambda}\left(\mu_{y}\left(\lambda^{\prime}, \lambda\right) \mu_{x}\left(\lambda^{\prime \prime}, \lambda\right)-\mu_{x}\left(\lambda^{\prime}, \lambda\right) \mu_{y}\left(\lambda^{\prime \prime}, \lambda\right)\right) \psi_{\lambda}^{2}
$$

Nous pouvons alors réécrire $J_{B}(f, g)$ sous la forme suivante

$$
J_{B}(f, g)=\sum_{\left(\lambda^{\prime}, \lambda^{\prime \prime}\right) \in \Lambda^{2}} 2^{j^{\prime}} \alpha_{\lambda^{\prime}} 2^{j^{\prime \prime}} \beta_{\lambda^{\prime \prime}} p_{\gamma_{2}}\left(\lambda^{\prime}, \lambda^{\prime \prime}\right) m_{\lambda^{\prime} \lambda^{\prime \prime}}
$$

Les deux lemmes suivants nous permettent alors de conclure. 
Lemme 2.1. La suite $\left(2^{j^{\prime}+j^{\prime \prime}} \alpha_{\lambda^{\prime}} \beta_{\lambda^{\prime \prime}} p_{\gamma_{2}}\left(\lambda^{\prime}, \lambda^{\prime \prime}\right)\right)_{\left(\lambda^{\prime}, \lambda^{\prime \prime}\right) \in \Lambda^{2}}$ appartient à l'espace $\ell^{1}\left(\Lambda^{2}\right)$.

Lemme 2.2. L'ensemble des $\left.\left(m_{\lambda^{\prime}, \lambda^{\prime \prime}}\right)_{\left(\lambda^{\prime}, \lambda^{\prime \prime}\right.}\right) \in \Lambda^{2}$ décrit une famille normalisée en norme $L^{1}$ de molécules.

Nous montrerons seulement ici pourquoi $m_{\lambda^{\prime} \lambda^{\prime \prime}}$ est une fonction de $L^{1}\left(\mathbb{R}^{2}\right)$, d'intégrale nulle.

Calculons tout d'abord, pour $\left(\lambda^{\prime}, \lambda^{\prime \prime}\right) \in \Lambda^{2}$, la somme

$$
\begin{aligned}
\sum_{\lambda \in \Lambda} \int_{\mathbb{R}^{2}}\left(\left|\mu_{y}\left(\lambda^{\prime}, \lambda\right) \mu_{x}\left(\lambda^{\prime \prime}, \lambda\right)-\mu_{x}\left(\lambda^{\prime}, \lambda\right) \mu_{y}\left(\lambda^{\prime \prime}, \lambda\right)\right| \psi_{\lambda}^{2}(x)\right) d x \\
\leq c 2^{j^{\prime}+j^{\prime \prime}} \sum_{\lambda \in \Lambda} p_{\gamma_{1}}\left(\lambda^{\prime}, \lambda\right) p_{\gamma_{1}}\left(\lambda^{\prime \prime}, \lambda\right) \\
\leq c\left(n, \gamma_{1}\right) 2^{j^{\prime}+j^{\prime \prime}} p_{\gamma_{1}}\left(\lambda^{\prime}, \lambda^{\prime \prime}\right)<+\infty
\end{aligned}
$$

Nous sommes donc en mesure d'intégrer terme à terme la série qui définit $m_{\lambda^{\prime} \lambda^{\prime \prime}}$

$$
\int m_{\lambda^{\prime} \lambda^{\prime \prime}}=\frac{2^{-\left(j^{\prime}+j^{\prime \prime}\right)}}{p_{\gamma_{2}}\left(\lambda^{\prime}, \lambda^{\prime \prime}\right)} \sum_{\lambda \in \Lambda}\left(\mu_{y}\left(\lambda^{\prime}, \lambda\right) \mu_{x}\left(\lambda^{\prime \prime}, \lambda\right)-\mu_{x}\left(\lambda^{\prime}, \lambda\right) \mu_{y}\left(\lambda^{\prime \prime}, \lambda\right)\right) .
$$

Or

$$
\begin{aligned}
\sum_{\lambda \in \Lambda}\left(\mu_{y}\right. & \left.\left(\lambda^{\prime}, \lambda\right) \mu_{x}\left(\lambda^{\prime \prime}, \lambda\right)-\mu_{x}\left(\lambda^{\prime}, \lambda\right) \mu_{y}\left(\lambda^{\prime \prime}, \lambda\right)\right) \\
& =\sum_{\lambda \in \Lambda}\left(\left\langle\frac{\partial \psi_{\lambda^{\prime}}}{\partial y}, \psi_{\lambda}\right\rangle\left\langle\frac{\partial \psi_{\lambda^{\prime \prime}}}{\partial x}, \psi_{\lambda}\right\rangle-\left\langle\frac{\partial \psi_{\lambda^{\prime}}}{\partial x}, \psi_{\lambda}\right\rangle\left\langle\frac{\partial \psi_{\lambda^{\prime \prime}}}{\partial y}, \psi_{\lambda}\right\rangle\right)
\end{aligned}
$$

et cette dernière série vaut exactement

$$
\left\langle\frac{\partial \psi_{\lambda^{\prime}}}{\partial y}, \frac{\partial \psi_{\lambda^{\prime \prime}}}{\partial x}\right\rangle-\left\langle\frac{\partial \psi_{\lambda^{\prime}}}{\partial x}, \frac{\partial \psi_{\lambda^{\prime \prime}}}{\partial y}\right\rangle=\int J\left(\psi_{\lambda^{\prime}}, \psi_{\lambda^{\prime \prime}}\right)=0 .
$$

\section{Généralisations.}

Nous présenterons par la suite des généralisations possibles aux résultats ci-dessus. 
3.1. Le cadre $L^{p}\left(\mathbb{R}^{n}\right) \times L^{q}\left(\mathbb{R}^{n}\right)$.

Le même opérateur $P^{\sharp}$ défini en Section 1.2 s'étend en un opérateur continu de $L^{p}\left(\mathbb{R}^{n}\right) \times L^{q}\left(\mathbb{R}^{n}\right)$ dans l'espace de Hardy $\mathcal{H}^{r}\left(\mathbb{R}^{n}\right)$, où $1<p<$ $+\infty, 1<q<+\infty, 1 / p+1 / q=1 / r$, à condition que $1 \geq r>n /(n+1)$.

Remarque. Cette restriction sur $r$ est due au manque d'oscillations des produits $\varphi_{j k} \psi_{j l}$ qui apparaissent dans la construction de $P^{\sharp}$. Cette limitation disparaît si nous considérons des renormalisations du produit obtenues à partir de symboles bilinéaires que nous définissons dans [7] et $[8]$.

La différence $P^{\sharp}-\tilde{P}^{\sharp}$ sera un opérateur borné de $L^{p}\left(\mathbb{R}^{n}\right) \times L^{q}\left(\mathbb{R}^{n}\right)$ dans l'espace de Lizorkin-Triebel homogène $\dot{F}_{r}^{0,1}\left(\mathbb{R}^{n}\right)$ avec les mêmes conditions sur $p, q$ et $r$.

Nous ne donnons pas ici la définition ni les propriétés des espaces de Lizorkin-Triebel; le lecteur intéressé pourra consulter [9] à ce sujet. Remarquons tout simplement que, si $r=1$, l'espace $\dot{F}_{1}^{0,1}$ coïncide avec l'espace de Besov homogène $\dot{B}_{1}^{0,1}$, et que, si $0<r \leq 1$, on a les inclusions strictes $\dot{F}_{r}^{0,1} \subset \mathcal{H}^{r}$.

Le théorème du Jacobien se généralise alors immédiatement au cadre $L^{p}\left(\mathbb{R}^{2}\right) \times L^{q}\left(\mathbb{R}^{2}\right)$ : si $\nabla f \in L^{p}\left(\mathbb{R}^{2}\right), \nabla g \in L^{q}\left(\mathbb{R}^{2}\right)$, alors $J(f, g) \in$ $\mathcal{H}^{r}\left(\mathbb{R}^{2}\right)$ pour $1<p<+\infty, 1<q<+\infty, 1 / r=1 / p+1 / q$, pourvu que $1 \geq r>2 / 3$.

Signalons maintenant que cette restriction $r>n /(n+1)$ dans l'énoncé du théorème du Jacobien n'est plus une limitation technique due à notre méthode, mais une limitation qui découle du fait que $J(f, g)$ n'a que son intégrale nulle, alors que les moments d'ordre supérieur ne le sont pas.

REMARQUes. La définition du produit renormalisé que nous proposons ici dépend du cadre fonctionnel dans lequel nous nous situons. Si on voulait, par exemple, trouver un "produit renormalisé" dans le cadre $L^{2} \times \mathrm{BMO}$, on ne devrait alors conserver que le terme $\sum_{j \in \mathbb{Z}}\left(P_{j} f\right)\left(Q_{j} g\right)$. 


\subsection{Application à d'autres opérateurs bilinéaires.}

Nous obtenons également des résultats analogues pour des opérateurs plus généraux.

Nous établissons, à titre d'exemple, le résultat suivant

Théorème 3.1. Soient $\left(A_{i}\right)_{i=1, K}$ et $\left(B_{i}\right)_{i=1, K}$ des opérateurs de Calderón-Zygmund qui vérifient les conditions

$$
A_{i}(1)=A_{i}^{*}(1)=B_{i}(1)=B_{i}^{*}(1)=0 \quad \text { pour tout } i=1, K
$$

Supposons que pour tout couple $(u, v)$ de fonctions de $L^{2}\left(\mathbb{R}^{n}\right)$ la fonction $\sum_{i=1}^{K} A_{i}(u) B_{i}(v)$ est d'intégrale nulle.

Alors l'opérateur bilinéaire $B$ défini par

$$
B(f, g)=\sum_{i=1}^{K} A_{i}(f) B_{i}(g)
$$

est continu de $L^{p}\left(\mathbb{R}^{n}\right) \times L^{q}\left(\mathbb{R}^{n}\right)$ dans l'espace de Hardy $\mathcal{H}^{r}\left(\mathbb{R}^{n}\right), 1<$ $p<+\infty, 1<q<+\infty, 1 / r=1 / p+1 / q$, pourvu que $1 \geq r>n /(n+1)$.

De façon plus précise, l'opérateur $B$ se décompose en $B=B_{1}+B_{2}$, où $B_{1}$ est continu de $L^{p}\left(\mathbb{R}^{n}\right) \times L^{q}\left(\mathbb{R}^{n}\right)$ dans $\mathcal{H}^{r}\left(\mathbb{R}^{n}\right)$ et $B_{2}$ l'est de $L^{p}\left(\mathbb{R}^{n}\right) \times L^{q}\left(\mathbb{R}^{n}\right)$ dans $\dot{F}_{r}^{0,1}\left(\mathbb{R}^{n}\right)$.

Remarque. Dans le cas particulier des opérateurs de convolution de Calderón-Zygmund, ce résultat a déjà été obtenu par R. Coifman et L. Grafakos ([2], [10]).

Ces idées de la preuve sont essentiellement les mêmes que pour le théorème du Jacobien.

Si $U$ est un opérateur de Calderón-Zygmund vérifiant $U(1)=$ $U^{*}(1)=0$, et si l'on pose $u\left(\lambda, \lambda^{\prime}\right)=\left\langle U\left(\psi_{\lambda}\right), \psi_{\lambda^{\prime}}\right\rangle$ pour $\left(\psi_{\lambda}\right)_{\lambda \in \Lambda}$ base orthonormée d'ondelettes, la matrice $\left(u\left(\lambda^{\prime}, \lambda\right)\right)_{\left(\lambda^{\prime}, \lambda\right) \in \Lambda^{2}}$ a une décroissance à partir de la diagonale donnée par l'estimation

$$
\left|u\left(\lambda, \lambda^{\prime}\right)\right| \leq c p_{\gamma}\left(\lambda, \lambda^{\prime}\right)
$$

où $p_{\gamma}$ est défini par $(1.22)$ et $\gamma>0$ dépend de la régularité de la base d'ondelettes. 
Pour effectuer la décomposition de l'opérateur bilinéaire $B$ en $B_{1}+$ $B_{2}$, rappelons que, $A_{i}$ et $B_{i}$ étant des opérateurs bornés dans $L^{2}$ (ou bien $L^{p}$ et $L^{q}$ ) on peut écrire

$$
B(f, g)=\sum_{i=1}^{K} P^{\sharp}\left(A_{i} f, B_{i} g\right)+\sum_{i=1}^{K} S\left(A_{i} f, B_{i} g\right)
$$

où

$$
S\left(A_{i} f, B_{i} g\right)=\sum_{\lambda}\left\langle A_{i} f, \psi_{\lambda}\right\rangle\left\langle B_{i} g, \psi_{\lambda}\right\rangle \psi_{\lambda}^{2} .
$$

Si on pose $B_{1}(f, g)=\sum_{i=1}^{K} P^{\sharp}\left(A_{i} f, B_{i} g\right), B_{1}$ a les propriétés annoncées.

Etudions maintenant $B_{2}(f, g)=\sum_{i=1}^{K} S\left(A_{i} f, B_{i} g\right)$. Posons, pour $i=1, K$,

$$
\begin{aligned}
a_{i}\left(\lambda^{\prime}, \lambda\right) & =\left\langle A_{i} \psi_{\lambda^{\prime}}, \psi_{\lambda}\right\rangle, \\
b_{i}\left(\lambda^{\prime \prime}, \lambda\right) & =\left\langle B_{i} \psi_{\lambda^{\prime \prime}}, \psi_{\lambda}\right\rangle .
\end{aligned}
$$

Le terme $B_{2}(f, g)$ s'écrit alors, si $f=\sum_{\lambda} \alpha_{\lambda} \psi_{\lambda}, g=\sum_{\lambda} \beta_{\lambda} \psi_{\lambda}$

$$
\begin{aligned}
B_{2}(f, g) & =\sum_{i=1}^{K} \sum_{\lambda}\left(\sum_{\lambda^{\prime}} \alpha_{\lambda^{\prime}} a_{i}\left(\lambda^{\prime}, \lambda\right)\right)\left(\sum_{\lambda^{\prime \prime}} \beta_{\lambda^{\prime \prime}} b_{i}\left(\lambda^{\prime \prime}, \lambda\right)\right) \psi_{\lambda}^{2} \\
& =\sum_{\lambda \in \Lambda} \sum_{\left(\lambda^{\prime}, \lambda^{\prime \prime}\right) \in \Lambda^{2}} \alpha_{\lambda^{\prime}} \beta_{\lambda^{\prime \prime}}\left(\sum_{i=1}^{K} a_{i}\left(\lambda^{\prime}, \lambda\right) b_{i}\left(\lambda^{\prime \prime}, \lambda\right)\right) \psi_{\lambda}^{2} .
\end{aligned}
$$

L'appartenance de $B_{2}(f, g)$ à $\dot{B}_{1}^{0,1}\left(\mathbb{R}^{n}\right)$ (respectivement $\dot{F}_{r}^{0,1}\left(\mathbb{R}^{n}\right)$ ) découle alors d'une démonstration analogue à celle du Théorème 2.2.

Remarquons seulement que

$$
\begin{aligned}
\int B_{2}(f, g) & =\sum_{\lambda} \sum_{\lambda^{\prime}} \sum_{\lambda^{\prime \prime}} \alpha_{\lambda^{\prime}} \beta_{\lambda^{\prime \prime}} \sum_{i=1}^{K} a_{i}\left(\lambda^{\prime}, \lambda\right) b_{i}\left(\lambda^{\prime \prime}, \lambda\right) \\
& =\sum_{\lambda^{\prime}} \sum_{\lambda^{\prime \prime}} \alpha_{\lambda^{\prime}} \beta_{\lambda^{\prime \prime}} \sum_{\lambda} \sum_{i=1}^{K}\left\langle A_{i} \psi_{\lambda^{\prime}}, \psi_{\lambda}\right\rangle\left\langle B_{i} \psi_{\lambda^{\prime \prime}}, \psi_{\lambda}\right\rangle \\
& =\sum_{\lambda^{\prime}} \sum_{\lambda^{\prime \prime}} \alpha_{\lambda^{\prime}} \beta_{\lambda^{\prime \prime}} \sum_{i=1}^{K} \int\left(A_{i} \psi_{\lambda^{\prime}}\right)\left(B_{i} \psi_{\lambda^{\prime \prime}}\right)=0
\end{aligned}
$$

$\operatorname{car} \int B\left(\psi_{\lambda^{\prime}}, \psi_{\lambda^{\prime \prime}}\right)=0$ par hypothèse. 


\section{Appendice. Lien entre renormalisation par ondelettes et renormalisation par paraproduit.}

Nous établirons ici le lien entre la renormalisation par ondelettes du produit, et d'autres méthodes de renormalisation. Dans le but d'alléger les notations, nous considérons par la suite le cas de la dimension 1.

Soit $\varphi$ une fonction appartenant à la classe de Schwartz telle que

$$
\left\{\begin{array}{l}
\hat{\varphi} \in \mathcal{D}(\mathbb{R}), \\
\hat{\varphi} \quad \text { est une fonction réelle et paire, } \\
\hat{\varphi}(\xi) \in[0,1], \text { pour tout } \xi \in \mathbb{R}, \\
\hat{\varphi}(\xi)=1 \text { si }|\xi| \leq \pi-\delta \quad(\delta<\pi / 3), \\
\hat{\varphi}(\xi)=0 \text { si }|\xi|>\pi+\delta, \\
\hat{\varphi}^{2}(\xi)+\hat{\varphi}^{2}(2 \pi-\xi)=1 \text { si } 0 \leq \xi \leq 2 \pi .
\end{array}\right.
$$

Pour tout $j \in \mathbb{Z}$ on désigne par $S_{j}$ l'opérateur de convolution avec $2^{j} \varphi\left(2^{j} x\right)$, et on pose $\Delta_{j}=S_{j+1}-S_{j}$.

Le paraproduit de J.M. Bony [1] est défini, pour $f$ et $g$ dans $L^{2}$, par

$$
\Pi(f, g)=\sum_{j \in \mathbb{Z}} S_{j-1}(f) \Delta_{j}(g) .
$$

REMARQue. La définition du paraproduit dépend du choix de la fonction $\varphi$; mais deux choix différents pour cette fonction conduisent en fait à des paraproduits "équivalents".

Il est bien connu que le paraproduit est un opérateur continu de $L^{2} \times L^{2}$ dans l'espace de Hardy $\mathcal{H}^{1}$.

Le produit de $f$ et de $g$ peut s'écrire, à l'aide du paraproduit,

$$
f g=\Pi(f, g)+\Pi(g, f)+\sum_{j \in \mathbb{Z}}\left(\Delta_{j} f\right)\left(\Delta_{j} g\right) .
$$

La définition de renormalisation au moyen du paraproduit surgit alors tout naturellement; on pose

$$
\Pi^{\sharp}(f, g)=\Pi(f, g)+\Pi(g, f)=f g-\sum_{j \in \mathbb{Z}}\left(\Delta_{j} f\right)\left(\Delta_{j} g\right) .
$$


Nous obtenons le résultat suivant

Théorème 4.1. Soit $\left(\psi_{\lambda}\right)_{\lambda \in \Lambda}$ une base orthonormée d'ondelettes. $L$ 'opérateur $P^{\sharp}-\Pi^{\sharp}$ envoie continûment $L^{2} \times L^{2}$ dans l'espace de Besov homogène $\dot{B}_{1}^{0,1}$.

L'invariance "modulo $\dot{B}_{1}^{0,1}$ " de la renormalisation du produit par rapport au choix de la base orthonormée d'ondelettes nous permet de considérer le cas de l'analyse multi-résolution dite de Littlewood-Paley. Dans ce cas $\varphi$ est la fonction définie par (4.1) et l'ondelette $\psi$ est définie par

$$
\hat{\psi}(\xi)=\left(\hat{\varphi}(\xi / 2)^{2}-\hat{\varphi}(\xi)^{2}\right)^{1 / 2} e^{-i \xi / 2} .
$$

Pour $f$ et $g$ dans $L^{2}(\mathbb{R})$, il s'agit de montrer que

$$
\pi_{1}(f, g)+\pi_{2}(f, g)+\pi_{3}(f, g)-\Pi(f, g)-\Pi(g, f) \in \dot{B}_{1}^{0,1}(\mathbb{R}) .
$$

Or on sait déjà que $\pi_{3}(f, g) \in \dot{B}_{1}^{0,1}(\mathbb{R})$. Finalement, par symétrie, il suffit de montrer que

$$
\sum_{j \in \mathbb{Z}}\left(P_{j} f\right)\left(Q_{j} g\right)-\sum_{j \in \mathbb{Z}}\left(S_{j-1} f\right)\left(\Delta_{j} g\right) \in \dot{B}_{1}^{0,1}(\mathbb{R}) .
$$

Cette preuve se décompose en trois étapes.

Lemme 4.1. Soient $f$ et $g$ deux fonctions de $L^{2}(\mathbb{R})$. La différence

$$
\Delta_{1}(f, g)=\sum_{j \in \mathbb{Z}}\left(P_{j} f\right)\left(Q_{j} g\right)-\sum_{j \in \mathbb{Z}}\left(P_{j-1} f\right)\left(Q_{j} g\right)
$$

appartient à l'espace de Besov homogène $\dot{B}_{1}^{0,1}(\mathbb{R})$.

La preuve de ce lemme est immédiate, car $\Delta_{1}$ s'écrit

$$
\Delta_{1}(f, g)=\sum_{j \in \mathbb{Z}}\left(Q_{j-1} f\right)\left(Q_{j} g\right) .
$$

Lemme 4.2. Soient $f$ et $g$ deux fonctions de $L^{2}(\mathbb{R})$. La différence

$$
\Delta_{2}(f, g)=\sum_{j \in \mathbb{Z}}\left(P_{j-1} f\right)\left(Q_{j} g\right)-\sum_{j \in \mathbb{Z}}\left(S_{j-1} f\right)\left(Q_{j} g\right)
$$

appartient à l'espace de Besov homogène $\dot{B}_{1}^{0,1}(\mathbb{R})$. 
La preuve repose sur une utilisation soigneuse de la structure particulière de l'analyse multirésolution de Littlewood-Paley. Remarquons tout d'abord que, si $\mathcal{F}$ désigne la transformée de Fourier, on a

$$
\mathcal{F}\left(V_{j}\right)=\left\{m\left(\xi / 2^{j}\right) \hat{\varphi}\left(\xi / 2^{j}\right): m \in L^{2}(0,2 \pi), m(\xi+2 \pi)=m(\xi)\right\} .
$$

Si, pour chaque $T>0$, on pose

$$
E_{T}=\left\{f \in L^{2}(\mathbb{R}): \operatorname{supp} \hat{f} \subset[-T, T]\right\},
$$

alors on a

$$
E_{(\pi-\delta) 2^{j}} \subset V_{j} \subset E_{(\pi+\delta) 2^{j}} .
$$

Nous effectuons maintenant une décomposition de la projection $P_{j} f$, pour chaque valeur de $j$ fixée,

$$
P_{j} f=P_{j} f_{1}^{j}+P_{j} f_{2}^{j}+P_{j} f_{3}^{j},
$$

où $f_{1}^{j}, f_{2}^{j}$ et $f_{3}^{j}$ sont définies par les conditions

$$
\left\{\begin{array}{l}
\operatorname{supp} \hat{f}_{1}^{j} \subset\left\{\xi \in \mathbb{R}:|\xi| \leq(\pi-\delta) 2^{j}\right\}, \\
\operatorname{supp} \hat{f}_{2}^{j} \subset\left\{\xi \in \mathbb{R}:(\pi-\delta) 2^{j} \leq|\xi| \leq(\pi+\delta) 2^{j}\right\}, \\
\operatorname{supp} \hat{f}_{3}^{j} \subset\left\{\xi \in \mathbb{R}:|\xi| \geq(\pi+\delta) 2^{j}\right\}, \\
f=f_{1}^{j}+f_{2}^{j}+f_{3}^{j} .
\end{array}\right.
$$

Dans ces conditions, on a $f_{1}^{j} \in E_{(\pi-\delta) 2^{j}} \subset V_{j}$, d'où $P_{j} f_{1}^{j}=f_{1}^{j}$. La fonction $f_{3}^{j}$ est orthogonale à $V_{j}$, d'où $P_{j} f_{3}^{j}=0$. Quant à $f_{2}^{j}$, elle est, par construction, orthogonale à $E_{(\pi-\delta) 2^{j}} \subset V_{j}$. En conséquence, sa projection $P_{j} f_{2}^{j}$ sera orthogonale à $E(\pi-\delta) 2^{j}$, et le spectre de $P_{j} f_{2}^{j}$ contenu dans la couronne

$$
\mathcal{C}_{j}=\left\{\xi \in \mathbb{R}:(\pi-j) 2^{j} \leq|\xi| \leq(\pi+j) 2^{j}\right\} .
$$

Si on calcule maintenant $S_{j} f$ à l'aide de cette même décomposition de $f$, on obtient

$$
S_{j} f_{1}^{j}=f_{1}^{j}, \quad S_{j} f_{3}^{j}=0, \quad \operatorname{supp} \widehat{f_{2}^{j}} \subset \mathcal{C}_{j}
$$

Finalement, pour chaque valeur de $j$ on a

$$
\left(P_{j}-S_{j}\right) f=\left(P_{j}-S_{j}\right) f_{2}^{j},
$$


et le spectre de $\left(P_{j}-S_{j}\right) f$ est contenu dans la couronne $\mathcal{C}_{j}$.

Quant au spectre de $Q_{j} g$, il est contenu, d'après (4.5) et (4.1) dans la couronne $\mathcal{D}_{j}=\left\{\xi \in \mathbb{R}:(\pi-\delta) 2^{j} \leq|\xi| \leq 2^{j+1}(\pi+\delta)\right\}$.

Ainsi, pour chaque valeur de $j$, le produit $\left(P_{j-1}-S_{j-1}\right)(f) Q_{j}(g)$ a son spectre contenu dans la couronne

$$
\left\{\xi \in \mathbb{R}:(\pi-3 \delta) 2^{j} \leq|\xi| \leq 5(\pi+\delta) 2^{j}\right\} .
$$

Ceci permet d'appliquer un lemme de "presque orthogonalité" et d'estimer

$$
\begin{aligned}
\| \sum_{j \in \mathbb{Z}}\left(P_{j-1}-S_{j-1}\right)(f) & Q_{j}(g) \|_{\dot{B}_{1}^{0,1}} \\
& \leq c \sum_{j \in \mathbb{Z}}\left\|\left(P_{j-1}-S_{j-1}\right)(f) Q_{j}(g)\right\|_{L^{1}(\mathbb{R})} \\
& \leq c\left(\sum_{j \in \mathbb{Z}}\left\|\left(P_{j}-S_{j}\right) f\right\|_{2}^{2}\right)^{1 / 2}\left(\sum_{j \in \mathbb{Z}}\left\|Q_{j} g\right\|_{2}^{2}\right)^{1 / 2} \\
& \leq c\|f\|_{2}\|g\|_{2} .
\end{aligned}
$$

Lemme 4.3. Soient $f$ et $g$ deux fonctions de $L^{2}(\mathbb{R})$. La différence

$$
\Delta_{3}(f, g)=\sum_{j \in \mathbb{Z}}\left(S_{j-1} f\right)\left(Q_{j} g\right)-\sum_{j \in \mathbb{Z}}\left(S_{j-1} f\right)\left(\Delta_{j} g\right)
$$

appartient à l'espace de Besov homogène $\dot{B}_{1}^{0,1}(\mathbb{R})$.

L'idée de départ est de réécrire

$$
\Delta_{3}(f, g)=\sum_{j \in \mathbb{Z}} S_{j-1}(f)\left(P_{j+1}-P_{j}\right)(g)-\sum_{j \in \mathbb{Z}} S_{j-1}(f)\left(S_{j+1}-S_{j}\right)(g),
$$

et d'appliquer, à chacune des séries ci-dessus, la transformation d'Abel.

Finalement

$$
\Delta_{3}(f, g)=\sum_{j \in \mathbb{Z}}\left(\Delta_{j-1} f\right)\left(S_{j+1} g-P_{j+1} g\right) .
$$

La fin de la démonstration est identique à celle du Lemme 4.2. 
Signalons finalement que nous avons developpé dans [7] et [8] la construction d'une renormalisation du produit associée à des symboles bilinéaires du type de ceux que R. Coifman et Y. Meyer ont considérés dans [3]; la renormalisation par paraproduit n'est qu'un cas particulier de cette renormalisation par symboles bilinéaires.

Modulo $\dot{B}_{1}^{0,1}\left(\dot{F}_{r}^{0,1}\right)$, toutes les méthodes de renormalisation que nous définissons sont équivalentes.

\section{References.}

[1] Bony, J. M., Calcul symbolique et propagation des singularités pour les E.D.P. non linéaires. Ann. Sci. Ecole Norm. Sup. 14 (1981), 209-246.

[2] Coifman, R. et Grafakos, L., Hardy space estimates for multilinear operators. Revista Mat. Iberoamericana 8 (1992), 45-67.

[3] Coifman, R. et Meyer, Y., Au delà des opérateurs pseudodifférentiels. Astérisque $\mathbf{5 7}$ (1978).

[4] Coifman, R. et Meyer, Y., Ondelettes et opérateurs III. Hermann, 1991.

[5] Coifman, R., Lions, P. L., Meyer, Y. et Semmes, S., Compensated compactness and Hardy space. J. Math. Pures et Appl. 72 (1993), 247-286.

[6] Daubechies, I., Orthonormal basis of compactly supported wavelets. Comm. Pure Appl. Math. 41 (1988), 909-996.

[7] Dobyinsky, S., Ondelettes, renormalisations du produit et applications à certains opérateurs bilinéaires. Thèse du CEREMADE, 1992.

[8] Dobyinsky, S., Lemme Div-Curl et renormalisations du produit. $J$. Math. Pures et Appl. 72 (1993), 239-245.

[9] Frazier, M., Jawerth, B. et Weiss, G., Littlewood-Paley theory and the study of functions spaces, à paraitre. CBMS-AMS Regional Conference series.

[10] Grafakos, L., Hardy space estimates for miltilinear operators, II. Revista Mat. Iberoamericana 8 (1992), 69-92.

[11] Mallat, S., Multiresolution approximation and wavelets. Technical Report, GRASP Lab., Dept. of Computer and Information Science, Univ. of Pensylvania.

[12] Meyer, Y., Ondelettes et fonctions splines. Technical Report, Séminaire E.D.P., Ecole Polytechnique, Paris, 1986.

[13] Meyer, Y., Ondelettes et opérateurs, I, Hermann, 1990.

[14] Meyer, Y., Ondelettes et opérateurs, II, Hermann, 1990. 
[15] Meyer, Y., La minimalité de l'espace de Besov $\dot{B}_{1}^{0,1}\left(\mathbb{R}^{n}\right)$ et la continuité des opérateurs définis par des intégrales singulières. Univ. Autónoma de Madrid, Monografías de Mat. 4, 1986.

Recibido: 16 de septiembre de 1.993

Revisado:

Sylvia Dobyinsky CEREMADE

Université Paris IX-Dauphine Place de Lattre de Tassigny 75775 Paris Cedex 16, FRANCE dobyinsk@modalx.u-paris10.fr 